\title{
La atencion a los crónicos pluripatologicos y ancianos en la era post-covid-19
}

\author{
Care of chronic pluri-pathological and aged patients \\ in the post-COVID-19 era
}

\author{
Antonio Pose Reino ${ }^{1}$, Javier Suarez Dono² \\ ${ }_{1,2}$ Unidad de medicina interna y pluripatologia. Servicio de medicina interna. Complexo Hospitalario Universitario de Santiago de Compostela y Barbanza. \\ ${ }^{\top}$ Director de la cátedra de cronicidad de la USC
}

Si hablamos de cronicidad en este momento de Pandemia, tenemos que darnos cuenta de que este concepto, tal y como lo teníamos concebido hasta el año 2020, ha cambiado de forma significativa.

La Pandemia ha hecho que aparezcan nuevos grupos de crónicos. Uno es el de personas que estaban sanas y que tras sufrir la COVID-19, han quedado crónicamente enfermas. Algunos autores consideran que podrían llegar a ser el 30\% de los que padecen la enfermedad y precisan ingreso, aunque también los que no precisaron ingreso pueden quedar con secuelas, que se harán crónicas. En este último caso, generalmente serán menos graves ${ }^{1-3}$.

Otro grupo es el de personas crónicas previas a la Pandemia que han sufrido la enfermedad y que se transformarán en pacientes crónicos complejos por las secuelas que les ha dejado la enfermedad en un aparato o sistema ya dañado por una enfermedad crónica, como puede ser la EPOC, la Insuficiencia cardíaca, la cirrosis hepática, el deterioro cognitivo, etc...

El tercer grupo es el de personas crónicas que se han dejado de evaluar de forma continuada durante este período, por las necesidades asistenciales impuestas por la Pandemia y que ha hecho que sus enfermedades evolucionen a formas más complejas, que requieren una mayor atención y conllevan un peor pronóstico. Esto incluye a personas con Cáncer cuyo diagnóstico se ha demorado. La Asociación española contra el Cáncer considera que el número de diagnósticos de cánceres nuevos disminuyó un 21\% durante el confinamiento. Esto es un reflejo de lo que puede suponer la Pandemia para la atención a los pacientes con Cáncer. Según un trabajo publicado recientemente en el British Medical Journal ${ }^{4}$, las personas cuyo tratamiento oncológico se retrasa incluso solo un mes, tienen un riesgo de morir entre un 6-13\% más alto y su riesgo sigue aumentando conforme se demora el tratamiento.

El cuarto grupo es el de personas sanas y que no han contraido la enfermedad, pero a las que las medidas aplicadas para controlar la Pandemia, le han dejado secuelas de aislamiento, soledad, depresión, deterioro cognitivo, trastornos psíquicos, etc...Estas medidas han afectado de forma muy especial a los más frágiles, más ancianos y que viven aislados, pero también han hecho mella en los más jóvenes.

Durante este período de Pandemia los Servicios de Medicina de Familia, Interna y de Neumología además de las UCls y Unidades de Reanimación, fueron los responsables de la atención directa a los pacientes COVID en la primera línea y esto ha hecho que los crónicos pluripatológicos que vienen siendo seguidos de forma continuada en nuestros servicios de Medicina Interna, se resientan. Sirva como ejemplo nuestra actividad en el hospital de día de pluripatología (HDD), que se ha visto reducida de forma significativa en el último año, muy especialmente durante los meses del confinamiento.

Igualmente en los 5 sectores de Medicina Interna, en los que tenemos dividida el área sanitaria de Santiago de Compostela, que establecen una estrecha relación con Primaria a través de la consulta electrónica y presencial y que atienden una población media de 66.000 personas por Sector y cerca de 20.000 mayores de 65 años por cada uno, con un $8 \%$ de mayores de 80 años y 50 médicos de Familia; hemos notado que la Pandemia nos ha afectado. En los meses de marzo y hasta finales de mayo del 2020 se ha producido un descenso del 30-50\% en Consultas, incluidas las e-consultas y con cifras similares en las atenciones del HDD. Esta tendencia se ha mantenido los dos primeros meses de este año en curso.

Si miramos a los pacientes ingresados en nuestro Servicio también aquí hemos notado una disminución, a pesar de que más del $80 \%$ de nuestros pacientes ingresan por urgencias y que por tanto su ingreso no se debería afectar por la Pandemia, pero la realidad es que ha habido un efecto disuasorio y muchos de los pacientes que tendrían que acudir a Urgencias para ingresar no lo hicieron.

Todas estas situaciones que hemos vivido durante el año 2020 y lo que va del 2021, harán que la visión de la Medicina Interna cambie. La figura del internista cobra especial relevancia ante las dificultades, por su versatilidad y capacidad de adaptación. Por otro lado, situaciones como la pandemia COVID, nos han puesto de manifiesto que es necesario un enfoque 


\section{"Todas estas situaciones que hemos vivido durante el año 2020 y lo que va del 2021, harán que la visión de la Medicina Interna cambie. La figura del internista cobra especial relevancia ante las dificultades, por su versatilidad y capacidad de adaptación"}

integral del paciente y un abordaje multidisciplinar que debe ser coordinado por un médico con capacidad de integrar todas estas medidas necesarias para el manejo de cada paciente.

La Medicina Interna no es una especialidad dedicada solo a tratar a las personas mayores, ni solo a los crónicos; pero tenemos que entender, porque los hechos nos lo hacen ver, que los crónicos, mayores y pluripatológicos son los pacientes más vistos en nuestros Servicios y cada vez más en todos los servicios de los hospitales. Por esto se hace necesaria una actitud proactiva con estos pacientes y no esperar, como hacemos hasta el momento a que el paciente acuda a urgencias cuando se descompense y tengamos que ingresarlo, con el deterioro funcional y psíquico que conlleva cada ingreso y que muchas veces se hace irreversible, con deterioro de la calidad de vida y aumento de la mortalidad.

El paciente crónico y pluripatológico es un paciente que debe ser tratado y seguido de forma continuada por un equipo multidisciplinar en Primaria, pero en íntimo contacto con el hospital, para tratar de resolver problemas puntuales y dudas que se generan en su atención y de cuya resolución puede depender el que un paciente ingrese o no y de que su calidad de vida se mantenga o no durante más tiempo.

En este número de la revista se publican dos artículos, uno de los cuales nos pone de relieve la importancia de las comorbilidades y la pluripatología en uno de los procesos más prevalentes en nuestros Servicios, como es la insuficiencia cardiaca. En el otro artículo se nos muestra las diferencias en las características de los pacientes mayores que residen en los centros sociosanitarios y los que permanecen en el núcleo familiar.

El primero es un estudio observacional y prospectivo, en el que se incluyeron 246 pacientes con insuficiencia cardíaca vistos en la Consulta de Medicina Interna de un hospital de tercer nivel, de los que 118 (48\%) fueron pluripatológicos (PP), siendo mayores los PP (77 vs 73 años). El artículo pone de manifiesto, la importancia de la pluripatología entre nuestros pacientes con insuficiencia cardíaca y que se trata de pacientes muy frágiles, aún no siendo todos muy mayores y que requieren un abordaje multidisciplinar e integral de su proceso.

El segundo es un estudio observacional, retrospectivo, que incluye 254 personas ancianas ingresadas en un Servicio de Medicina Interna, con una media de edad próxima a los 80 años y de las que la mayoría 78,7\%, viven todavía en sus residencias familiares. Los pacientes que vivían en su casa familiar eran más independientes, aunque tenían el mismo número de comorbilidades, si bien los que no vivían en el domicilio familiar tenían más patología neurológica. Es de destacar que hubo más fallecimientos tras el alta, en este grupo.

Como se desprende de ambos artículos y enlazando con lo que comentábamos al principio de esta editorial, los pacientes que ingresan en los Servicios de Medicina Interna son cada vez más mayores y con más comorbilidades y peor pronóstico. El manejo en la Planta de hospitalización durante las descompensaciones, no es una medida eficiente para mejorar el pronóstico ni la calidad de vida de estas personas. Es necesario implantar de forma progresiva un sistema integrado, continuado, proactivo, compartido con primaria y que nos permita un enfoque multidisciplinar, individualizado y ambulatorio; adecuando las prestaciones a las necesidades del paciente. Estas prestaciones tendrán que ponerse a disposición del individuo, de forma precoz, en el ámbito donde se encuentra, evitando los ingresos hospitalarios, que lejos de mejorar el pronóstico, deterioran de forma progresiva la calidad de vida de nuestros pacientes crónicos.

\section{BIBLIOGRAFÍA}

1. Del Rio C, Collins LF, Malani P. Long-term health consequences of COVID-19. JAMA. 2020;324(17):1723-1724

2. Carfi A, Bernabei R, Landi F, Gemelli Against COVID-19 Post-Acute Care Study Group. Persistent symptoms in patients after acute COVID-19. JAMA. 2020;324(6):603-605.

3. Logue JK, Franko NM, McCulloch DJ, McDonald D, Magedson A, Wolf CR. Sequelae in Adults at 6 Months After COVID-19 Infection. JAMA Network Open. 2021; 4(2): e210830.

4. P Hanna TP, KingWD, Thibodeau S, Jalink M, Paulin GA, Harvey-Jones E, et al. Mortality due to cancer treatment delay: systematic review and meta-analysis. BMJ 2020; 371: m4087.

5. "Marques MR, Gonçalves H, Calvao J, Gairigolzarri J, Mendez M, Bover R, Vilacosta I, Macaya C. La pluripatologia en una Unidad de Insuficiencia Cardíaca: Ia perspectiva de un internista. Galicia Clin 2021; 82-2: 81-86"

6. "Azevedo $S$, Maia R, Guerreiro E. Ancianos: relación entre residencia y estado de salud. Galicia Clin 2021; 82-2: 75-80" 